\title{
A Comparative Study of Electron Energy-Loss Spectroscopy (EELS) and X-ray Absorption Near Edge Structure (XANES) Spectroscopy for Probing the Oxidation State of Transition Metals in Planetary Materials
}

\author{
Diana Bolser ${ }^{1}$ and Thomas Zega ${ }^{2}$ \\ ${ }^{1 .}$ University of Arizona, Department of Chemistry and Biochemistry, 1306 E. University Blvd., Tucson \\ AZ 85721 \\ 2. University of Arizona, Lunar and Planetary Laboratory, 1629 E. University Blvd., Tucson AZ 85721.
}

Planetary materials contain an abundance of $3 d$ transition metals. They occur in mineral phases that formed in a wide range of environments, e.g., the gaseous envelopes surrounding ancient stars and the early solar nebula. The ability of such metals to occur in multiple oxidation states makes them potentially useful recorders of the redox conditions under which their host assemblage formed or last equilibrated. Thus, measurements of the oxidation state of $3 d$ metals in planetary materials can serve as a probe of redox conditions in ancient nebular or stellar environments. Such measurements are important for characterizing returned samples from current and future missions, e.g., Stardust [1] and OSIRIS-Rex.

Perovskite $\left(\mathrm{CaTiO}_{3}\right)$ is the second phase after corundum that is thermodynamically predicted to condense from a gas of solar composition [2]. It is an important constituent of calcium-aluminum-rich inclusions (CAIs) as well as isolated grains in the fine-grained matrix that pervades these primitive rocks. Given the range of conditions potentially experienced by CAIs, such as condensation, large-scale radial transport in the solar nebula, and hydrothermal alteration on parent bodies [3], we hypothesize that the $\mathrm{Ti}$ in CAI perovskite can occur in multiple valence states. Indeed, Ti and $\mathrm{V}$ have already been found in multiple oxidation states in hibonite and pyroxene in CAIs [4-5]. Here we present a study on the oxidation state of $\mathrm{Ti}$ in perovskite using electron energy-loss spectroscopy (EELS) and X-ray absorption near edge structure (XANES) spectroscopy.

We measured a combination of synthetic perovskite prepared under controlled oxygen fugacity $\left(f \mathrm{O}_{2}\right)$ conditions and meteoritic perovskite extracted from a CAI identified in the Axtell CV3 chondrite (AX30, Smithsonian). The perovskite grain was extracted and thinned to electron and X-ray transparency with an FEI Nova 200 focused-ion-beam scanning-electron-microscope. The synthetic perovskite was sintered in air at $1530^{\circ} \mathrm{C}$ for 48 hours. Electron microprobe scans detected no impurities and stoichiometric Ti content. From this batch, aliquots were removed for treatment in a gas-mixing furnace. One such sample was equilibrated under highly reducing conditions $\left(1200^{\circ} \mathrm{C}\right.$ and $\left.\log f \mathrm{O}_{2}=-20\right)$ for 121 hours.

For EELS analysis we used the $100 \mathrm{keV}$ monochromated and aberration-corrected Nion UltraSTEM at Arizona State University. XANES spectroscopy was performed using the scanning transmission X-ray microscope (STXM) on beamline 5.3.2 at the Advanced Light Source (ALS), Lawrence Berkeley National Laboratory. The UltraSTEM is capable of sub-Ångstrom spatial resolution and $\leq 0.03 \mathrm{eV}$ spectral resolution while STXM is capable of $\approx 30 \mathrm{~nm}$ spatial resolution and $0.1 \mathrm{eV}$ spectral resolution. High spectral resolution is needed to reveal the near-edge structure and chemical shift that is fundamental to measuring changes in oxidation state. In EELS and XANES, the L ionization edges of transition metal elements display sharp peaks at the near-edge region due to electronic transitions from $2 p$ core levels to empty $3 d$ states [6]. A 
change in oxidation state causes a chemical shift in the spectrum from lower to higher energies as the valence of the metal changes from reduced to oxidized states [7]. While both techniques now provide similar spectral resolution, STXM is likely to provide superior signal-to-noise ratio, whereas the UltraSTEM can provide atomically resolved information on oxidation state.

XANES spectra of the $\mathrm{Ti} \mathrm{L}_{2,3}$ absorption edges for synthetic perovskites show a chemical shift between the reduced and as-grown perovskites. The reduced sample has an edge onset $0.3 \mathrm{eV}$ lower in energy than the as-grown sample, consistent with a decrease in the oxidation state of $\mathrm{Ti}$ atoms (Fig. 1). The spectrum of a perovskite grain extracted from a CAI shows a less pronounced pre-edge region that more closely resembles the as-grown sample, suggesting a predominant $\mathrm{Ti}^{4+}$ component. A partially resolved shoulder on the second L3 peak appears in the spectrum of meteoritic perovskite. The cause of this feature has been attributed to distortions in the octahedral coordination environment of $\mathrm{Ti}$ atoms [8]. Complementary EELS measurements of as-grown perovskite show the same transitions (Fig. 2). A more detailed comparison of the obtained spectra will be presented at the meeting.

Previous studies have demonstrated the utility of EELS and XANES for analysis of the oxidation states of $3 d$ transition metals in planetary materials [9-11]. These spectroscopic methods are important for sample-return missions, e.g., Stardust, and will continue to be important for future analyses of returned samples from Hayabusa-2 and OSIRIS-REx. However, the sample requirements for EELS versus STXM are more stringent. The former requires very thin $(<100 \mathrm{~nm})$ samples, which will necessitate the optimization of FIB-based preparation techniques in achieving desired thicknesses and minimizing surface damage. STXM is much more forgiving of variations in sample thickness, but if atomic-scale information is important, then STEM-based EELS measurements will be required.

\section{References:}

[1] M Chi et al., GCA 73 (2009) 7150-7161.

[2] DS Ebel MESS II (2006), 253-277.

[3] AN Krot et al., Meteorit Planet Sci 30 (1995), 748-775.

[4] JR Beckett et al., GCA 52 (1988) 1479-1495.

[5] SB Simon et al., GCA 71 (2007), 3098-3118.

[6] R Brydson, EMSA Bulletin 21 (1991), 57-67.

[7] PA van Aken et al., J Phys Condens Mat 25 (1998), 323-327.

[8] FMF de Groot, Phys Rev B 42 (1990), 5459-5468.

[9] LP Keller and S. Messenger, GCA 75, (2011), 5336-5365.

[10] MS Thompson and TJ Zega, LPSC 45 (2014), 2834.

[11] TJ Zega et al., Am Min 88 (2003) 1169-1172.

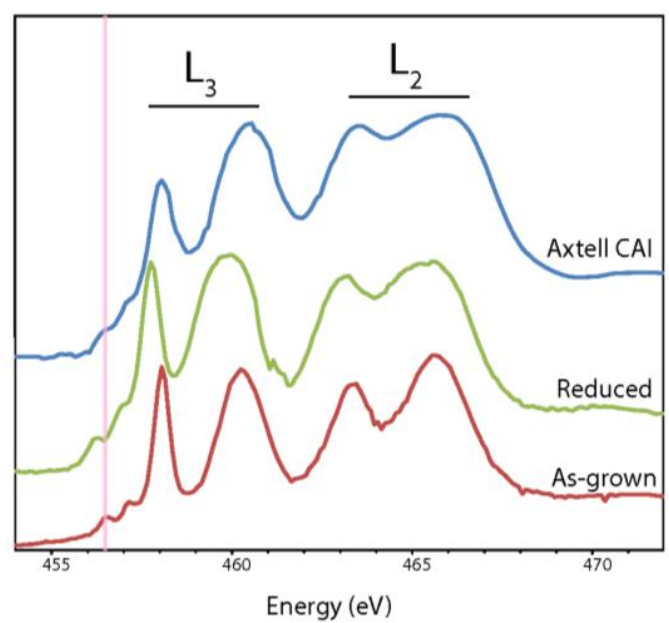

Figure 1 Ti $\mathrm{L}_{2,3}$ XANES spectra of (top) a perovskite grain extracted from Axtell; (middle) synthetic perovskite equilibrated at $1200^{\circ} \mathrm{C}$ and $\log f \mathrm{O}_{2}=$ -20 for 121 hours; (bottom) perovskite synthesized in air at $1530^{\circ} \mathrm{C}$. The shaded line indicates edge onset for the as-grown sample. Edge onset for the reduced sample is shifted $0.3 \mathrm{eV}$ lower.

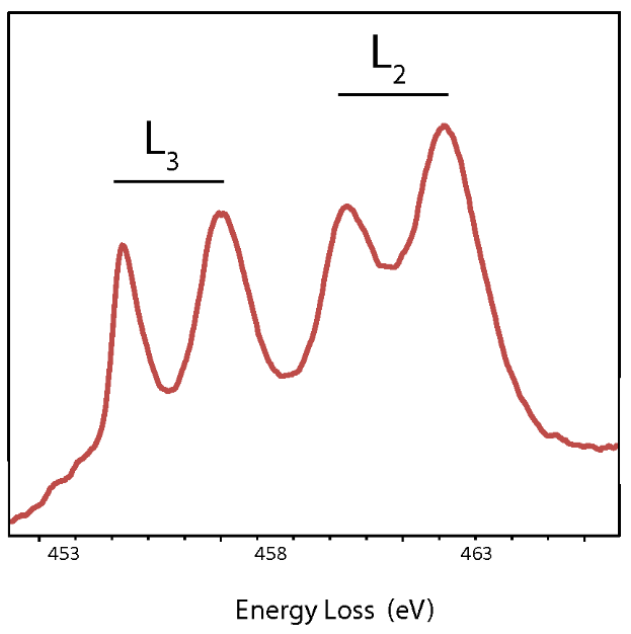

Figure $2 \mathrm{Ti} \mathrm{L}_{2,3}$ EELS spectrum of synthetic perovskite acquired using a $1.2 \AA$ probe and a $1 \mathrm{~mm}$ spectrometer entrance aperture. 